\title{
Malaria Control and Elimination: How Far we are: An Opinion Article
}

\section{Siddiqui FA*}

Department of Entomology, Pennsylvania State University, USA

*Corresponding author: Siddiqui FA, Department of Entomology, Pennsylvania State University, 546 Ag Sciences \& Industries Bldg, University Park, PA 16802, USA, Tel: +1 814-865-4700; E-mail: fas5130@psu.edu

Received date: Oct 10, 2016, Accepted date: Oct 13, 2016, Published date: Oct 15, 2016

Copyright: ( 2016 Siddiqui FA. This is an open-access article distributed under the terms of the Creative Commons Attribution License, which permits unrestricted use, distribution, and reproduction in any medium, provided the original author and source are credited.

Citation: Siddiqui FA (2016) Malaria Control and Elimination: How Far we are: An Opinion Article. J Biom Biostat 7: 321. doi:10.4172/2155-6180.1000321

\section{Malaria Control and Elimination}

Malaria is caused by four Plasmodium species in humans $(P$. falciparum, $P$. vivax, $P$. malariae and $P$. ovale) which spread from one person to another via the bite of female Anopheles mosquito. $P$. falciparum causes most deaths from malaria and is most prevalent on the African continent whereas $P$. vivax has a wider geographical distribution. According to the latest WHO estimates, released in December 2015, there were 214 million cases of malaria in 2015 and 438,000 deaths. Existing strategies to control malaria include vector control, chemoprevention and case management [1]. There are currently no licensed vaccines against malaria. RTS, S/AS01 is the only ongoing research vaccine against $P$. falciparum, which might still take 3 to 5 years to come out, if safety and effectiveness are considered acceptable. Thus, in absence of a successful vaccine, malaria control relies on the use of anti-malarial drugs. Artemisinin-based combination therapies (ACTs) are the best available treatment for $P$. falciparum malaria. The impact of the use of ACTs for malaria treatment is proven by the fact that Tu Youyou was awarded the 2015 Nobel Prize for Medicine for the discovery of the malaria drug, Artemisinin. But to the dismay of malaria research community all over the world, parasite resistance to artemisinins has been detected in 5 countries of the Greater Mekong subregion: Cambodia, Lao People's Democratic Republic, Myanmar, Thailand and Viet Nam as observed in case of all previous anti-malarial drugs. The major concern now is the spread of multi-drug resistance to other regions with dire public health consequences. The most critical benchmark in the study of ART resistance was the identification of single point mutations in the propeller region of $P$. falciparum kelch protein gene on chromosome 13 (PfK13) as a molecular marker associated with delayed parasite clearance in vitro and in vivo. Numerous studies have been reported to determine the levels of polymorphisms of $\mathrm{K} 13$ in this region in order to map the spread and evolution of ART resistance [2-4]. Very interestingly there have been some reports of slow parasite clearance rates even in absence of $\mathrm{K} 13$ mutant alleles suggesting the role of additional molecules in development of ART resistance in $P$. falciparum. It would be crucial to identify additional genetic loci involved in ART resistance.

The pace at which the geographical extent of artemisinin resistance is spreading is faster than the rate at which control and elimination measures are being developed and introduced. This emphasizes the fact that apart from understanding the current state and mechanisms of antimalarial drug resistance it is extremely essential at the same time to expand the current arsenal used against the parasite. This would include the identification and development of novel vaccine candidates and the anti-malarial drug targets for malaria [5-7].

\section{References}

1. World Health Organization (2015) World malaria report 2015. WHO.

2. Ménard D, Khim N, Beghain J, Adegnika AA, Shafiul-Alam M, et al. (2016) A Worldwide Map of Plasmodium falciparum K13-Propeller Polymorphisms. N Engl J Med 25:2453-64.

3. Wang M, Siddiqui FA, Fan Q, Luo E, Cao Y, et al. Limited Genetic Diversity in the PvK12 Kelch protein in Plasmodium vivax isolates from Southeast Asia.

4. Siddiqui FA, Wang M, Cui L, Artemisinin resistance and K 13-A Systemic Review.

5. Siddiqui FA, Dhawan S, Singh S, Singh B, Gupta P, et al. (2013) Functional Characterization of a Thrombospondin Structural Repeat Containing Rhoptry Protein from Plasmodium falciparum merozoites. Cell Microbiol.

6. Pandey AK, Reddy KS, Sahar T, Gupta S, Singh H, et al. (2012) Identification of a potent combination of key Plasmodium falciparum merozoite antigens that elicit strain transcending parasite neutralizing antibodies. Infect Immun 81: 441-451.

7. Antony HA, Parija SC (2016) Antimalarial drug resistance: An overview. Trop Parasitol. 6: 30-41. 